\title{
Differential relieving effects of shikonin and its derivatives on inflammation and mucosal barrier damage caused by ulcerative colitis
}

\author{
Hongwei Han ${ }^{\text {Equal first author, } 1,2}$, Wenxue Sun ${ }^{\text {Equal first author, } 1,2}$, Lu Feng ${ }^{1,2}$, Zhongling Wen ${ }^{1,2}$, Minkai Yang ${ }^{1,2}$, Yingying Ma ${ }^{1,2}$ \\ , Jiangyan $\mathrm{Fu}^{1,2}$, Xiaopeng Ma ${ }^{1,2}$, Xinhong $\mathrm{Xu}^{1,2}$, Zhaoyue Wang ${ }^{1,2}$, Tongming Yin ${ }^{2}$, Xiao-Ming Wang ${ }^{1,2}$, Gui-Hua \\ Lu $^{1,3}$, Jin-Liang Qi ${ }^{1,2}$, Hongyan Lin ${ }^{\text {Corresp., 1, } 2}$, Yonghua Yang ${ }^{\text {Corresp. 1, } 2}$ \\ ${ }^{1}$ State Key Laboratory of Pharmaceutical Biotechnology, Institute of Plant Molecular Biology, School of Life Sciences, Nanjing University, Nanjing, China \\ 2 Co-Innovation Center for Sustainable Forestry in Southern China, MOE Key Laboratory of Forest Genetics and Biotechnology, Nanjing Forestry University, \\ Nanjing, China \\ 3 School of Life Sciences, Huaiyin Normal University, Huaian, China \\ Corresponding Authors: Hongyan Lin, Yonghua Yang \\ Email address: linhy@nju.edu.cn, yangyh@nju.edu.cn
}

Background: Ulcerative colitis (UC) is one of the most challenging human diseases. Natural shikonin (SK) and its derivatives (with have higher accumulation) isolated from the root of Lithospermum erythrorhizon have numerous beneficial effects, such as wound healing and anti-inflammatory activities. Some researchers have reported that hydroxynaphthoquinone mixture (HM) and SK attenuate the acute UC induced by dextran sulfate sodium (DSS). However, no existing study has systemically investigated the effectiveness of SK and other hydroxynaphthoquinone natural derivative monomers on UC.

Methods: In this study, mice were treated with SK and its derivatives ( $25 \mathrm{mg} / \mathrm{kg}$ ) and mesalazine (200 $\mathrm{mg} / \mathrm{kg}$ ) after DSS administration daily for one week. Disease progression was monitored daily by observing the changes in clinical signs and body weight.

Results: Intragastric administration natural single naphthoquinone attenuated the malignant symptoms induced by DSS. SK or its derivatives remarkably suppressed the serum levels of pro-inflammatory cytokines while increasing the inflammatory cytokine interleukin (IL)-10. Additionally, both SK and alkanin restrained the activities of cyclooxygenase-2 (COX-2), myeloperoxidase (MPO) and inducible nitric oxide synthase (iNOS) in serum and colonic tissues. SK and its derivatives inhibited the activation of nucleotide binding oligomerization domain-like receptors (NLRP3) inflammasome and NF-KB signaling pathway, thereby relieving the DSS-induced disruption of epithelial tight junction (TJ) in colonic tissues.

Conclusions: Our findings shed more lights on the pharmacological efficacy of SK and its derivatives in UC against inflammation and mucosal barrier damage. 
1 Differential Relieving effects of shikonin and its derivatives on 2 inflammation and mucosal barrier damage caused by ulcerative colitis

3

4 Hongwei Han ${ }^{1,2 \dagger}$, Wenxue Sun ${ }^{1,2 \dagger}$, Lu Feng ${ }^{1,2}$, Zhongling Wen ${ }^{1,2}$, Minkai Yang ${ }^{1,2}$, Yingying Ma ${ }^{1,2}$,

5 Jiangyan $\mathrm{Fu}^{1,2}$, Xiaopeng $\mathrm{Ma}^{1,2}$, Xinhong $\mathrm{Xu}^{1,2}$, Zhaoyue Wang ${ }^{1,2}$, Tongming $\mathrm{Yin}^{2}$, Xiaoming Wang ${ }^{1,2}$,

6 Guihua $\mathrm{Lu}^{1,3}$, Jinliang Qi ${ }^{1,2}$, Hongyan Lin ${ }^{1,2^{*}}$, Yonghua Yang ${ }^{1,2^{*}}$

7

8 'State Key Laboratory of Pharmaceutical Biotechnology, Institute of Plant Molecular Biology,

9 School of Life Sciences, Nanjing University, Nanjing, PR China

${ }^{2}$ Co-Innovation Center for Sustainable Forestry in Southern China, MOE Key Laboratory of

Forest Genetics and Biotechnology, Nanjing Forestry University, Nanjing, PR China

${ }^{3}$ School of Life Sciences, Huaiyin Normal University, Huaian, PR China

$\dagger$ These authors contributed equally to this work

*Corresponding authors

Contact information of main corresponding author:

Dr. Yonghua Yang,

State Key Laboratory of Pharmaceutical Biotechnology, Nanjing University, Nanjing, PR China.

Tel/Fax: 86-25-89686305,

Email: yangyh@nju.edu.cn 


\section{Abstract}

36 Background: Ulcerative colitis (UC) is one of the most challenging human diseases. Natural

37 shikonin (SK) and its derivatives (with have higher accumulation) isolated from the root of

Lithospermum erythrorhizon have numerous beneficial effects, such as wound healing and anti-

inflammatory activities. Some researchers have reported that hydroxynaphthoquinone mixture

(HM) and SK attenuate the acute UC induced by dextran sulfate sodium (DSS). However, no

41 existing study has systemically investigated the effectiveness of SK and other

hydroxynaphthoquinone natural derivative monomers on UC.

Methods: In this study, mice were treated with SK and its derivatives $(25 \mathrm{mg} / \mathrm{kg})$ and mesalazine $(200 \mathrm{mg} / \mathrm{kg})$ after DSS administration daily for one week. Disease progression was monitored daily by observing the changes in clinical signs and body weight.

Results: Intragastric administration natural single naphthoquinone attenuated the malignant

47 symptoms induced by DSS. SK or its derivatives remarkably suppressed the serum levels of pro-

48 inflammatory cytokines while increasing the anti-inflammatory cytokine interleukin (IL)-10.

49 Additionally, both SK and alkanin restrained the activities of cyclooxygenase-2 (COX-2), myeloperoxidase (MPO) and inducible nitric oxide synthase (iNOS) in serum and colonic tissues. SK and its derivatives inhibited the activation of nucleotide binding oligomerization domain-like receptors (NLRP3) inflammasome and NF- $\kappa$ B signaling pathway, thereby relieving the DSS-induced disruption of epithelial tight junction (TJ) in colonic tissues.

54 Conclusions: Our findings shed more lights on the pharmacological efficacy of SK and its derivatives in UC against inflammation and mucosal barrier damage. 
59

\section{Introduction}

61 Inflammatory bowel disease (IBD), which encompasses Crohn's disease (CD) and ulcerative colitis (UC), is a kind of refractory and relapsing gastrointestinal inflammatory disease. IBD has not only affected the living quality of patients but also increased the risk of colon cancer if not treated in a timely manner (Ungaro et al., 2017; Stone et al., 2003). An increasing amount of evidence indicates that the incidence of colorectal cancer in IBD patients is generally higher than that in the general population (Terzic et al., 2010). Currently, traditional treatment strategies for IBD can be classified as three categories, namely, aminosalicylic acid agents, glucocorticoids, and immunosuppressive agents (Huang et al., 2009). Typically, 5-aminosalicylic acid remains the preferred drug in treating IBD, which can directly treat enteritis through inhibiting inflammatory reaction in the intestinal tract and exerting antibacterial function. Nonetheless, this drug will be quickly absorbed by the small intestine if taken orally, leading to acute and chronic renal injury. Moreover, the drug cannot reach the inflammatory site, which can hardly meet the treatment goal (Munk et al., 2004). In addition, glucocorticoid is likely to develop dependence after long-term application, and disease recurrence is common after drug withdrawal (Katz et al., 2004). By contrast, immunosuppressant is suitable for patients with chronic enteritis, which can inhibit lymphocyte proliferation, to achieve the therapeutic effect; nonetheless, it is associated with strong toxic side effects (Schroder et al., 2003). The emergence of anti-tumor necrosis factor therapy has made a major advance in the treatment of patients with IBD for the past two decades (Khan et al., 2019). However, despite the fact that this therapy is useful to over half of IBD patients, a substantial proportion of patients are primary nonresponders or lose response with long-term use (Hanauer et al., 2002; Ma et al., 2014). Moreover, this therapy causes some disturbing safety issues encompassing the increased risk of infections and malignant tumor in 
83 some groups (Lichtenstein et al., 2012). These factors are the reason for searching other safe,

84 mild, durable, and effective drugs to cure IBD.

85 The disease characteristics of IBD and shortcomings of current treatments forced us to turn 86 to the traditional Chinese medicine (TCM). Lithospermum erythrorhizon (L. erythrorhizon) is a 87 commonly used TCM in the Chinese pharmacopoeia of the People's Republic of China and is 88 typically abundant in shikonin (SK) derivatives, which have anti-tumor, anti-virus, and anti89 inflammatory activities (Chen et al., 2019; Yoshida et al., 2017). For example, Fan et al. reported 90 that the crude extract of Arnebia euchroma is effective on rats with experimental colitis (Fan et 91 al., 2013). Dozens of species of SK are available in the Boraginaceae plants, among which the 92 representative ones are SK, $\beta, \beta$-dimethylacryl-SK $(\beta, \beta$-dimethylacryl-SK), acetyl-SK (acetyl$93 \mathrm{SK}$ ), 5,8-dihydroxy-1, 4-naphthoquinone (naphthoquinone), and alkanin (AK, the enantiomeric 94 of SK) with high contents (Papageorgiou et al., 2006). They belong to the naphthoquinones that 95 are collectively known as SKs, which typically possess the advantages of bright color, long96 lasting effect, and nontoxic side effects. Currently, the efficacy of each individual component of 97 the crude extract has not been studied in detail. The pathological changes in the DSS-induced 98 IBD model are similar to human UC. Thus, it is an ideal model that has been widely used to 99 study the mechanism of UC and for screening potential drugs (Farooq et al., 2018).

100 In the present work, we successfully established a murine IBD model by treating C57BL/6 101 mice with DSS and the effectiveness of some natural SKs with high contents in L. erythrorhizon 102 were further studied and compared for their efficacy in IBD treatment to explore the potential 103 mechanisms. 


\section{Materials \& Methods}

108

109 Chemicals, Regents and antibodies

110 Chemicals and Regents were described in Supplementary File 1. All of compounds were

111 characterized by ${ }^{13} \mathrm{C}$ NMR, ${ }^{1} \mathrm{H}$ NMR in Supplementary File 2 . All antibodies are described in

112 Table S1. ECL Kit (\#34077) was purchased from Thermo Scientific (USA). Haematoxylin-

113 Eosin/HE Staining Kit (\#20170804) was purchased from Solarbio Science \& Technology Co.

114 Ltd (Beijing, China).

\section{Animals}

116 A total of 66 health male C57BL/6 mice weighted 18-20 g (6-8 weeks old) were selected for in 117 vivo experiment. All of them were purchased from Model Animal Research Center of Nanjing 118 University (Nanjing, China), All animal experiments and welfare were treated in strict 119 accordance with the relevant Guidelines for Care and Use of Laboratory Animals of Nanjing 120 University and approved by the Laboratory Animal Ethics Committee of School of Life 121 Sciences, Nanjing University (IACUC-1909002). The mice were group-housed in a laboratory 122 with controlled conditions $\left(22 \pm 2^{\circ} \mathrm{C}\right.$ and $60 \% \pm 5 \%$ humidity) under a $12 \mathrm{~h}$ light-dark cycle 123 throughout the experiment. Animals were supplied with standard diet and sterile water ad 124 libitum. All efforts were aimed at reducing the number and suffering of experimental animals 125 while meeting the needs of the experiment.

126

\section{DSS-induced colitis and design of drug treatment}

128 The mice were randomly divided into eight groups $(n=6$ per group) after one week of 129 acclimatization, including the model (the dextran sulfate sodium; 3.5\% (w/v) DSS), SK (6.25, $13012.5,25 \mathrm{mg} / \mathrm{kg}), \mathrm{AK}(25 \mathrm{mg} / \mathrm{kg})$, naphthoquinone (25 mg/kg), acetyl-SK (25 mg/kg), $\beta, \beta$ - 
131 dimethylacryl-SK (25 mg/kg), mesalazine (positive drug; 100, $200 \mathrm{mg} / \mathrm{kg}$ ) and control group,

132 then all the groups mice except for control group were exposed to $3.5 \%$ DSS, which was

133 dissolved in drinking water. All drugs were dissolved in olive oil and were perfused $200 \mu \mathrm{L}$ per

134 mice once daily at a predetermined dose by gavage which started at the same time as the DSS

135 treatment and continued one week. In the control and DSS-induced groups, the mice fed orally

136 with the same amount of olive oil. There was no significant difference in water intake among all

137 groups. Body weight and the disease activity index (DAI) were measured and recorded everyday

138 (Farooq et al., 2018). The DAI was calculated according to the standard listed in Table S2. After

139 one week of treatment, the entire mice monocular eyeball in each mouse was removed under

140 lightly anesthetized (carbon dioxide euthanasia) for blood collection. Then, all mice were

141 euthanized by cervical dislocation in the unconscious state. The colonic tissues were quickly and

142 safely removed and flushed with ice-cold PBS for pathological analysis. The same protocol was

143 carried out at least three times in independent experiments.

\section{Measurement of Cytokines, COX-2, MPO and iNOS in serum}

145 After drug treatment, mice were sacrificed at the end of the experiment. The whole bloods

146 samples were collected from peripheral blood after reperfusion. Afterwards, the blood was

147 allowed to clot by standing at room temperature for $30 \mathrm{~min}$, and later the clot was removed by

148 centrifuging at $2000 \mathrm{~g}$ for $10 \mathrm{~min}$ in a refrigerated centrifuge. The resultant supernatant (serum)

149 was then immediately transferred into a clean polypropylene tube. Kits were used to assay the

150 level of various cytokines (IL-6, IL-1 $\beta$, TNF- $\alpha$ and IL-10), COX-2, MPO and iNOS in the serum

151 of mice according to the product's guidelines (Yang et al., 2020).

152 Haematoxylin \& Eosin (H\&E) staining 
153 Small sections of colonic tissue were fixed in $10 \%$ buffered formalin and embedded in paraffin.

154 Thereafter, the sections were stained with haematoxylin and eosin (H\&E) and the colonic

155 mucosa was histologically evaluated by a pathologist in a blinded fashion, which was widely

156 used as evaluation criterion (Fan et al., 2013).

157 Immunohistochemical analysis

158 Immunohistochemistry was performed for Cyclooxygenase-2 (COX-2), NF- $\kappa B$, NLRP3, the 159 assay was conducted as described in (Xu et al., 2019). Briefly, the tissues were incubated with

160 primary antibodies: anti-NF- $\mathrm{B}$ p65 (1:100), anti-COX-2 (1:200) and anti-NLRP3 (1:100) at $4^{\circ} \mathrm{C}$

161 for at least $12 \mathrm{~h}$. Then, the sections were wash with PBS thrice and added with Alexa Fluor 488

162 labeled anti-mouse secondary antibody (Invitrogen, USA) at $25^{\circ} \mathrm{C}$ for $60 \mathrm{~min}$. Signals were 163 developed with Hematoxylin and DAB (Dako, Agilent Technologies, USA). Sections were 164 examined using light microscopy and image analysis software (Image-pro plus 6.0; Media

165 Cybernetics, Inc., Rockville, MD, USA) was used. The positive cumulative optical density (IOD) 166 and the tissue pixel AREA (AREA) of each photo were measured, and thus average optical value 167 (AO) was calculated by using $\mathrm{AO}=\mathrm{IOD} / \mathrm{AREA}$.

168 Western blot analysis

169 Colonic samples were lysed with RIPA lysis buffer which containing 1\% PMSF at 1:100 dilution 170 on ice for $30 \mathrm{~min}$. The insoluble components of cell lysates were removed by centrifugation 171 (10000 $\mathrm{rpm}$ at $4^{\circ} \mathrm{C}$ for $10 \mathrm{~min}$ ) and the harvested protein concentrations were assessed by using 172 the BCA Protein Assay kit. Then the following immunoblot analysis was referred to the study by 173 Farooq et al (Farooq et al., 2018). Finally, the immunoblots were quantified by densitometry with 174 Image J Software (National Institutes of Health, Bethesda, Maryland, USA).

\section{Data and Statistical analysis}


176 Results were presented as mean \pm S.E.M. of three independent experiments, with six mice $(n=6)$

177 per group to ensure their reliability. Statistical comparisons between the treated and untreated

178 groups were performed using Student's $t$-test (two-tailed) using GraphPad PRISM5. Differences

179 with a $P$-value $<0.05$ were considered statistically significant.

180

181

Results

182

SK and its derivatives protected the colon from dextran sulfate sodium (DSS)-induced

damage in an acute ulcerative colitics mouse model

184

An acute DSS-induced ulcerative colitis (UC) mouse model was established to investigate the

185

effects of SK and its derivatives on colitis. Mice were given either sterile water or $3.5 \%$ DSS

186

dissolved in sterile water in the model. Six other groups received 3.5\% DSS and were

187

administered SK (25 mg/kg), naphthoquinone (25 mg/kg), $\beta, \beta$-dimethylacryl-SK ( $25 \mathrm{mg} / \mathrm{kg})$,

acetyl-SK $(25 \mathrm{mg} / \mathrm{kg}), \mathrm{AK}(25 \mathrm{mg} / \mathrm{kg})$, and mesalazine $(200 \mathrm{mg} / \mathrm{kg})$ orally both at the beginning

189

of the experiment ( $n=6$ mice per group). SK and its derivatives could significantly attenuate the

190

severity level of inflammation at the end of this experiment. DSS group mice suffered a

191

shortening of about $30 \%$ compared with normal mice in the case of the colorectum length. SK

192

and its derivatives significantly prevented this shortening (Fig. 1A-1B). Moreover, SK and its

derivatives-treated mice showed improvements in body weight and disease activity index, as

194

well as the alleviation of splenomegaly (Fig. 1C-1F). Therefore, SK and its derivatives

195

ameliorated the colon syndromes of DSS-induced damage in mice.

SK and its derivatives ameliorated the DSS-induced histological damage

The colon tissues of mice in each group were stained with H\&E staining to observe the

pathological changes. The results (Fig. 2A) indicated that compared with other derivatives group,

inflammatory cells infiltration significantly decreased, the number of goblet cells decreased, 
200 andcrypt abscesses were distinct reduced in SK and AK-treated group. Grading was performed in

201 a blinded manner by a pathologist and the inflamation score result was showed in Fig. 2B. In

202 brief, the administration of SK and AK markedly relieved the symptoms of DSS-induced IBD in

203 mice.

204 SK and its derivatives relieved DSS-induced colonic epithelial TJ disruption in mice

205 Zonula occludens 1 (ZO-1), vascular cell adhesion molecule-1 (VCAM-1), Occludin and

206 Claudin-1 proteins play an important role in epithelial TJ, which can protect mucosa epithelial

207 cells from breaching by the harmful substances, maintain cellular permeability and integrity, and

208 ensure homeostasis of internal environment. Therefore, the effect of SK and its derivatives on

209 epithelial TJ proteins were further investigated. These results suggested that the expression of

210 ZO-1, VCAM-1, Occludin, and Claudin-1 were remarkably reduced in the DSS group compared

211 with the control group. However, SK and its derivatives at $25 \mathrm{mg} / \mathrm{kg}$ can restore the expression

212 of these epithelial TJ proteins (Fig. 3A-3B).

213 SK and its derivatives inhibit the activation of NLRP3 inflammasome in colonic tissues

214 Many studies have revealed that NLRP3 affects several inflammatory disorders to a large extent

215 including colitis (Lesuis et al., 2012). Fig. 4A-4B shows the significantly up-regulated expression

216 of NLRP3, apoptosis-associated speck-like protein containing a CARD (ASC), caspase-1, and

217 IL-1 $\beta$ in DSS group compared with control group. However, changes of caspase-1 and IL-1 $\beta$

218 were significantly reduced by SK and naphthoquinone. SK- and $\beta, \beta$-Dimethylacryl-SK-treament

219 groups evident reduced the level of ASC protein.

220 SK and its derivatives limited the expression of pro-inflammatory cytokines

221 When the circulating leukocytes are recruited into the colon, pro-inflammatory mediators are

222 released, including pro-inflammatory cytokines such as IL-6, IL-1 $\beta$ and TNF- $\alpha$. The levels of IL- 
2236 , IL-1 $\beta$, TNF- $\alpha$ and IL-10 were detected in the serum from 3.5\% DSS-exposed mice given

224 either control diet or SK and its derivatives to determine whether SK and its derivatives

225 attenuated the release of pro-inflammatory cytokines, thereby protecting the intestinal mucosa.

226 Fig. 5A-5D showed that treatment with SK and its derivatives at $25 \mathrm{mg} / \mathrm{kg}$ significantly reduced

227 the levels of pro-inflammatory cytokines (IL-6, IL-1 $\beta$ and TNF- $\alpha$ ) and increased the level of 228 anti-inflammatory cytokine (IL-10) in the serum. SK showed the best anti-inflammation effect 229 that was similar to mesalazine group at $200 \mathrm{mg} / \mathrm{kg}$. Fig. $5 \mathrm{E}-5 \mathrm{H}$ showed that $\mathrm{SK}$ and mesalazine 230 (the positive control) reduced the levels of pro-inflammatory cytokines (IL-6, IL-1 $\beta$ and TNF- $\alpha$ ) 231 and increased the level of anti-inflammatory cytokine (IL-10) in a dose-dependent manner, and 232 SK treatment resulted in improved anti-inflammatory effect at a dose of $25 \mathrm{mg} / \mathrm{kg}$. SK and its 233 derivatives may improve the severity of colon damage induced by DSS by inhibiting the 234 expression of pro-inflammatory cytokines.

\section{SK and its derivatives reduced the activities of MPO, COX-2 and iNOS in serum}

236 MPO is an enzyme that is largely present in neutrophils, and in monocytes and macrophages in 237 small concentrations (Davies et al., 2020) and the high levels of COX-2 (Chen et al., 2016) and 238 iNOS (Pandurangan et al., 2014) plays a central role in the initiation and propagation of the 239 inflammation. MPO, COX-2 and iNOS concentrations in all the mice groups are shown in Fig. 240 6A-6C. The MPO activity is an indicator of neutrophil infiltration in inflamed colon tissues. 241 Administration of DSS increased the activity of MPO as compared with normal mice. SK and 242 AK administration at $25 \mathrm{mg} / \mathrm{kg}$ markedly reduced the concentrations of MPO, COX-2 and iNOS 243 compared with DSS-induced mice.

244 SK and its derivatives attenuate the production of COX-2, NF-кB and NLRP3 in colonic 245 tissues 
246 Immunohistochemical expressions of COX-2, NF- $\mathrm{BB}$ and NLRP3 in all the groups of mice are 247 shown in Fig. 7A-7C. DSS administration caused the increased production of COX-2, NF- $\kappa$ B 248 and NLRP3 in colonic tissues compared with the control group. Usage of SK and its derivatives 249 reduced the expressions of COX-2, NF- $\mathrm{BB}$ and NLRP3 significantly compared with mice 250 induced with DSS.

251 SK and its derivatives regulate the COX-2 and iNOS expressions and the activation of NF$252 \kappa \mathbf{B}$ in colonic tissues

253 Transcription factors of the NF- $\mathrm{BB}$ family play a vital role in regulating genes, which can 254 influence the immune and inflammatory response (Andujar et al., 2012). Evidence has verified

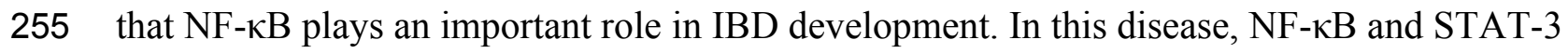
256 activation is observed in inflamed colonic mucosa, leading to the production of COX-2 and

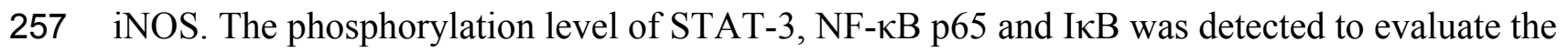
258 mechanism of SK. The outcome shown in Fig. 8A-8B suggested that the phosphorylation levels

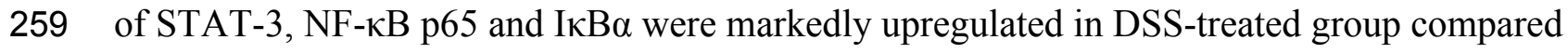
260 with the control group. These increases were markedly attenuated by treatment with DSS plus 261 SK and its derivatives at $25 \mathrm{mg} / \mathrm{kg}$, thereby decreasing the generation of COX-2 and iNOS in the 262 colon homogenates (Fig. 8C-8D).

263 Discussion

264 L. erythrorhizon is a kind of TCM used for wound healing in ancient times. Its roots are typically 265 rich with a variety of bioactive substances, including natural naphthoquinones, which represent 266 an ongoing source of therapeutic agents (Qiu et al., 2018). Among the naphthoquinones isolated 267 from L. erythrorhizon, SK has attracted extensive interest due to its multiple biological activities, 268 such as anti-inflammatory (Yoshida et al., 2017; Yang et al., 2014), wound healing (Yao et al., 
269 2019), anti-microbial (Andujar et al., 2013), anti-ulcer (Lesuis et al., 2012), anti-thrombus

270 (Andrikopoulos et al., 2003), and anti-cancer activities (Lin et al., 2018). Nearly 20 kinds of

271 natural SK derivatives have been identified.

272 The anti-inflammatory effects of natural naphthoquinones have been extensively

273 investigated. For instance, Fan et al. investigated the effectiveness of hydroxynaphthoquinone

274 mixture (HM) isolated from A. euchroma in rats with experimental colitis induced by 2, 4, 6-tri-

275 nitrobenzene sulfonic acid (TNBS) in 2013 (Fan et al., 2013). Their results suggested that HM

276 can remarkably attenuate the clinical and histopathological severity of the TNBS-induced colitis

277 in a dose-dependent manner. In addition, HM can also do the following: alleviate loss of body

278 weight, hematochezia, and inflammation; reduce the macroscopic damage score; and improve

279 the histological signs. Moreover, HM can evidently reduce the inflammatory infiltration, ulcer

280 area, and the severity of goblet cell depletion. Notably, HM, which is isolated from A. euchroma,

281 contains seven $\mathrm{AK}$ derivatives, including $\mathrm{AK}$, deoxyalkannin, acetylalkannin, $\beta, \beta$ -

282 dimethylacrylalkannin, $\alpha$-methylalkannin, isovalerylalkannin, and $\beta$-acetoxyisovalerylalkannin.

283 Isabel et al. reported that the oral administration of SK can attenuate the DSS-induced acute UC

284 by blocking the activation of two major targets, NF-кB and STAT-3 (Andujar et al., 2012).

285 However, the effectiveness of the commonly used SK and its natural derivatives on acute colitis

286 has not been systematically investigated yet.

287 In the present study, five representative natural naphthoquinones, namely, naphthoquinone,

$288 \mathrm{SK}$, acetyl-SK, $\beta, \beta$-dimethylacryl-SK and AK, were selected to treat DSS-induced colitis in

289 mice. The administration of these five natural naphthoquinones could ameliorate the clinical

290 severity of the wasting disease, prevent the shortening the length of colorectum, decrease the loss

291 of body weight, improve the appearance of feces, and prevent bloody excrements. Such 
292 beneficial effects were further proved by histological evaluation, as evidenced by the markedly

293 reduced severity and extent of inflamed tissue damage and infiltration of inflammatory cells.

294 Typically, the relative expression of pro-inflammatory cytokines, including IL-6, IL-1 $\beta$ and

295 TNF- $\alpha$ in serum in colon tissues, suggested that these five natural naphthoquinones had

296 beneficial effects. MPO is a marker of neutrophil infiltration, which has been observed to be

297 activated in several experimental colitis models, including the DSS-induced colitis. Therefore,

298 MPO is an indicator to quantify intestinal inflammation and evaluate the severity of

299 inflammation. In addition, the pro-inflammatory synthases, including iNOS and COX-2, are

300 inflammatory mediators with vital functions during the pathogenesis of UC (Itzkowitz et al.,

301 2006). The five natural naphthoquinones could suppress MPO, iNOS and COX-2 activities. Such

302 results were consistent with those found in histological examination, in which the inflammatory

303 extent in colonic tissues was less than that in SK- and AK-treated animals and naphthoquinone-,

304 acetyl-SK- and $\beta, \beta$-dimethylacryl-SK-treated mice. Moreover, results of western blot had further

305 verified that SK can relieve the colitis in mice by inhibiting the activation of NF- $\mathrm{kB}$ and NLRP3

306 inflammasome and disruption of epithelial TJ proteins. Collectively, these aforementioned

307 results offer insights into the potential use of SK and its derivatives in treating IBD.

308

309

310

311 Our results suggested that intra-gastric administration of single compound like SK or its

312 derivatives contributed to attenuating the loss of body weight, shortening the colon length,

313 increasing the spleen weight and relieving colonic epithelial tight disruption in mice induced by

314 DSS. Through a number of related pathological experiments, we have explained the underlying

315 mechanism that SK and its derivatives could inhibit the activation of NLRP3 inflammasome and 
$316 \mathrm{NF}-\kappa \mathrm{B}$ signaling pathway. These findings provide new evidence for the potential use of SK and

317 its derivatives to treat the inflammatory bowel disease (IBD).

318 Additional Information and Declarations

319

320

\section{Data Availability}

321 The following information was supplied regarding data availability:

322 The raw data are available in the Supplementary File 3. The raw data is showing H\&E data, IHC 323 raw data and Western blot bands.

324

325

\section{References}

326

327

Ungaro R, Mehandru S, Allen PB, Peyrin-Biroulet L, Colombel JF. 2017. Ulcerative colitis.

328

Lancet 359: 1756-1770 https://doi.org/ 10.1016/S0140-6736(16)32126-2.

Stone MA, Mayberry JF, Baker R. 2003. Prevalence and management of inflammatory bowel disease: a cross-sectional study from central England. European journal of gastroenterology \& hepatology 15:1275-1280 https://doi.org/ 10.1097/00042737-200312000-00004.

Terzic J, Grivennikov S, Karin E, Karin M. 2010. Inflammation and colon cancer.

Gastroenterology 138: 2101-2114 https://doi.org/ 10.1053/j.gastro.2010.01.058.

Huang LJ, Zhu Q, Lei M, Cao Q. 2009. Current use of immunosuppressive agents in inflammatory bowel disease patients in East China. World journal of gastroenterology 15:30553059 https://doi.org/10.3748/wjg. 15.3055.

Munk EM, Pedersen L, Floyd A, Norgard B, Rasmussen HH, Sorensen HT. 2004. pancreatitis: a population-based case-control study. The American journal of gastroenterology 
341 Katz JA. 2004. Treatment of inflammatory bowel disease with corticosteroids. Gastroenterology

342 clinics of North America 33:171-189 https://doi.org/ 10.1016/j.gtc.2004.02.002.

343 Schroder O, Stein J. 2003. Low dose methotrexate in inflammatory bowel disease: current

344 status and future directions. The American journal of gastroenterology 98:530-537

345 https://doi.org/ 10.1016/S0002-9270(02)06027-6.

346 Khan S, Rupniewska E, Neighbors M, Singer D, Chiarappa J, Obando C. 2019. Real-world

347 evidence on adherence, persistence, switching and dose escalation with biologics in adult

348 inflammatory bowel disease in the United States: A systematic review. Journal of clinical

349 pharmacy and therapeutics 44:495-507 https://doi.org/ 10.1111/jcpt.12830.

350 Hanauer SB, Feagan BG, Lichtenstein GR, Mayer LF, Schreiber S, Colombel JF,

351 Rachmilewitz D, Wolf DC, Olson A, Bao W, Rutgeerts P, Group AIS. 2002. Maintenance

352 infliximab for Crohn's disease: the ACCENT I randomised trial. Lancet 359:1541-1549

353 https://doi.org/ 10.1016/S0140-6736(02)08512-4.

354 Ma C, Huang V, Fedorak DK, Kroeker KI, Dieleman LA, Halloran BP, Fedorak RN. 2014.

355 Crohn's disease outpatients treated with adalimumab have an earlier secondary loss of response

356 and requirement for dose escalation compared to infliximab: a real life cohort study. Journal of

357 Crohn's \& colitis 8:1454-1463 https://doi.org/ 10.1016/j.crohns.2014.05.007.

358 Lichtenstein GR, Feagan BG, Cohen RD, Salzberg BA, Diamond RH, Price S, Langholff W,

359 Londhe A, Sandborn WJ. 2012. Serious infection and mortality in patients with Crohn's

360 disease: more than 5 years of follow-up in the TREAT registry. The American journal of

361 gastroenterology 107:1409-1422 https://doi.org/ 10.1038/ajg.2012.218.

362 Chen Y, Chen ZY, Chen L, Zhang JY, Fu LY, Tao L, Zhang Y, Hu XX, Shen XC. 2019.

363 Shikonin inhibits triple-negative breast cancer-cell metastasis by reversing the epithelial-to- 
364 mesenchymal transition via glycogen synthase kinase 3 beta-regulated suppression of beta-

365 catenin signaling. Biochemical pharmacology 166:33-45 https://doi.org/

$366 \quad 10.1016 /$ j.bcp.2019.05.001.

367 Yoshida LS, Kakegawa T, Yuda Y, Takano-Ohmuro H. 2017. Shikonin changes the

368 lipopolysaccharide-induced expression of inflammation-related genes in macrophages. Journal 369 of natural medicines 71:723-734 https://doi.org/ 10.1016/j.bcp.2019.05.001.

370 Fan HY, Zhang ZL, Liu K, Yang MY, Lv WH, Che X, Xu H, Song WW. 2013. Effectiveness

371 of a hydroxynaphthoquinone fraction from Arnebia euchroma in rats with experimental colitis.

372 World journal of gastroenterology 19:9318-9327 https://doi.org/ 10.3748/wjg.v19.i48.9318.

373 Papageorgiou VP, Assimopoulou AN, Samanidou VF, Papadoyannis IN. 2006. Analytical

374 methods for the determination of alkannins and shikonins. Current Organic Chemistry 10:583-

375622 https://doi.org/ 10.2174/138527206776055277.

376 Farooq SM, Hou Y, Li H, O'Meara M, Wang Y, Li C, Wang JM. 2018. Disruption of GPR35

377 Exacerbates Dextran Sulfate Sodium-Induced Colitis in Mice. Digestive diseases and sciences

378 63:2910-2922 https://doi.org/ 10.1007/s10620-018-5216-z.

379 Yang L, Zhou RY, Tong Y, Chen PF, Shen Y, Miao S, Liu XQ. 2020. Neuroprotection by

380 dihydrotestosterone in LPS-induced neuroinflammation. Neurobiology of Disease 140: 104814-

381 104827. https://doi.org/10.1016/j.nbd.2020.104814.

382 Xu M, Duan XY, Chen QY, Fan H, Hong ZC, Deng SJ, Nan Z, Wu H, Dong YL, Liu YJ,

383 Zhou CZ. 2019. Effect of compound sophorae decoction on dextran sodium sulfate (DSS)-

384 induced colitis in mice by regulating Th17/Treg cell balance. Biomedicine \& Pharmacotherapy

385 109:2396-2408 https://doi.org/ 10.1016/j.biopha.2018.11.087.

386 Andujar I, Rios JL, Giner RM, Miguel Cerda J, Recio Mdel C. 2012. Beneficial effect of 
387 shikonin on experimental colitis induced by dextran sulfate sodium in BALB/c mice. Evidence-

388 based complementary and alternative medicine : eCAM 2012:271606-271620 https://doi.org/

$38910.1155 / 2012 / 271606$.

390 Qiu HY, Wang PF, Lin HY, Tang CY, Zhu HL, Yang YH. 2018. Naphthoquinones: A

391 continuing source for discovery of therapeutic antineoplastic agents. Chemical biology \& drug

392 design 91:681-690 https://doi.org/ 10.1111/cbdd.13141.

393 Yang Y, Wang J, Yang Q, Wu S, Yang Z, Zhu H, Zheng M, Liu W, Wu W, He J, Chen Z.

394 2014. Shikonin inhibits the lipopolysaccharide-induced release of HMGB1 in RAW264.7 cells

395 via IFN and NF-kappaB signaling pathways. International immunopharmacology 19:81-87

396 https://doi.org/ 10.1016/j.intimp.2014.01.003.

397 Yao CH, Chen KY, Chen YS, Li SJ, Huang CH. 2019. Lithospermi radix extract-containing

398 bilayer nanofiber scaffold for promoting wound healing in a rat model. Materials science \&

399 engineering C, Materials for biological applications 96:850-858 https://doi.org/

$400 \quad$ 10.1016/j.msec.2018.11.053.

401 Andujar I, Rios JL, Giner RM, Recio MC. 2013. Pharmacological Properties of Shikonin - A

402 Review of Literature since 2002. Planta Medica 79:1685-1697 https://doi.org/ 10.1055/s-0033-

4031350934.

404 Lesuis N, Befrits R, Nyberg F, van Vollenhoven RF. 2012. Gender and the treatment of

405 immune-mediated chronic inflammatory diseases: rheumatoid arthritis, inflammatory bowel

406 disease and psoriasis: an observational study. BMC Medicine 10:82-90. https://doi.org/

$407 \quad 10.1186 / 1741-7015-10-82$.

408 Davies MJ, Hawkins CL. 2020. The Role of Myeloperoxidase in Biomolecule Modification, 409 Chronic Inflammation, and Disease. Antioxid Redox Sign. 32: 957-981. 10.1089/ars.2020.8030. 
410 Chen L, Teng H, Fang T, Xiao J. 2016. Agrimonolide from Agrimonia pilosa

411 suppresses inflammatory responses through down-regulation of COX-2/iNOS and

412 inactivation of NF- $\mathrm{BB}$ in lipopolysaccharide-stimulated macrophages. Phytomedicine

413 23:846-855. https://doi.org/10.1016/j.phymed.2016.03.016.

414 Pandurangan AK, Kumar SA, Dharmalingam P, Ganapasam S. 2014. Luteolin, a 415 bioflavonoid inhibits azoxymethane-induced colon carcinogenesis: involvement of iNOS and 416 COX-2. Pharmacogn. Mag. 10: 306-310. https:// doi.org/10.4103/0973-1296.133285.

417 Andrikopoulos NK, Kaliora AC, Assimopoulou AN, Papapeorgiou VP. 2003. Biological 418 activity of some naturally occurring resins, gums and pigments against in vitro LDL oxidation. 419 Phytotherapy Research 17:501-507 https://doi.org/ 10.1002/ptr.1185.

420 Lin HY, Han HW, Sun WX, Yang YS, Tang CY, Lu GH, Qi JL, Wang XM, Yang YH. 2018. 421 Design and characterization of alpha-lipoic acyl shikonin ester twin drugs as tubulin and PDK1 422 dual inhibitors. European journal of medicinal chemistry 144:137-150 https://doi.org/ 423 10.1016/j.ejmech.2017.12.019.

424 Itzkowitz SH. 2006. Molecular biology of dysplasia and cancer in inflammatory bowel disease. 425 Gastroenterology clinics of North America 35:553-571 https://doi.org/ 10.1016/j.gtc.2006.07.002. 426 
Figure 1

SK andits derivatives protect the colon from DSS-induced damage

(A) Macroscopic observation of colon length. (B) Bar graph showing colon length. ${ }^{* *} p<0.01$ versus control group. (C) Pattern of daily weight changes. $* p<0.05,{ }^{* *} p<0.01$ versus DSS-treated group. (D) Disease activity index of mice in each group. ${ }^{*} p<0.05,{ }^{* *} p<0.01$ versus DSS-treated group. (E) Macroscopic observation of spleen size. (F) Bar graph showing spleen weight. Data are shown as mean \pm S.E.M, ${ }^{*} p<0.05$ versus control group ( $n=6$ per group).

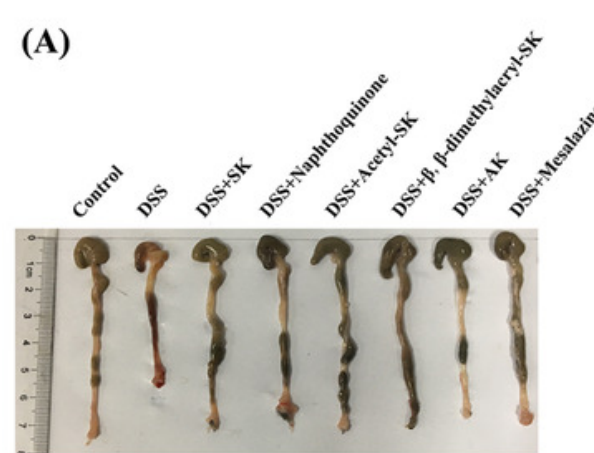

(B)

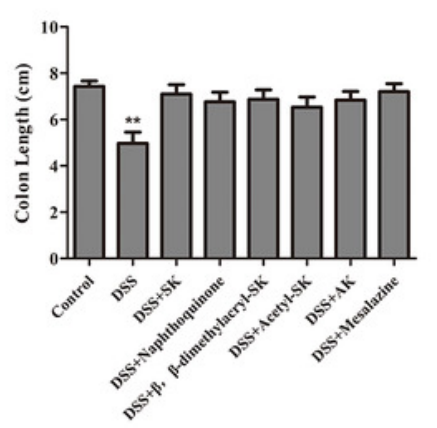

(C)
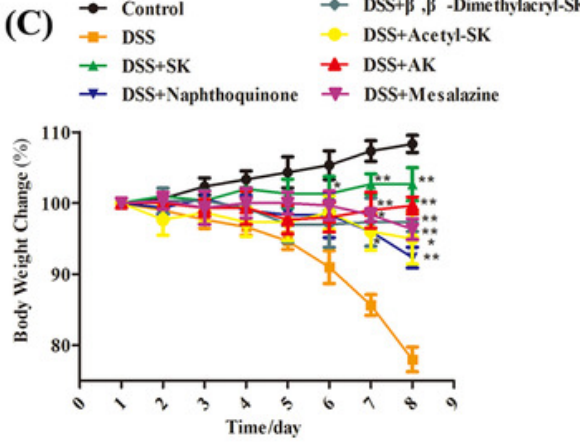

(D)

(D) $\rightarrow$ Control - DSS * DSS+Naphthoquinone $\rightarrow$ DSS+Mesalazine

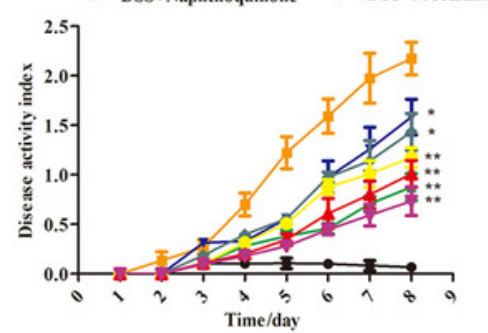

(E)
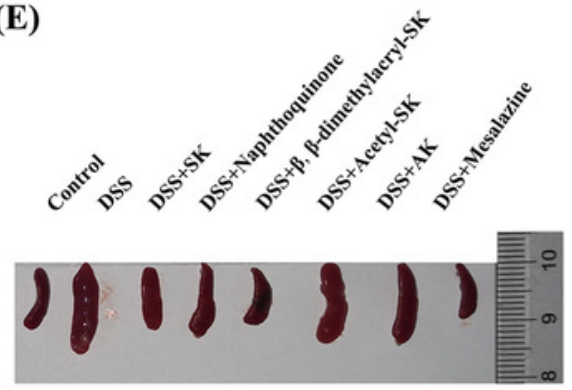

(F)

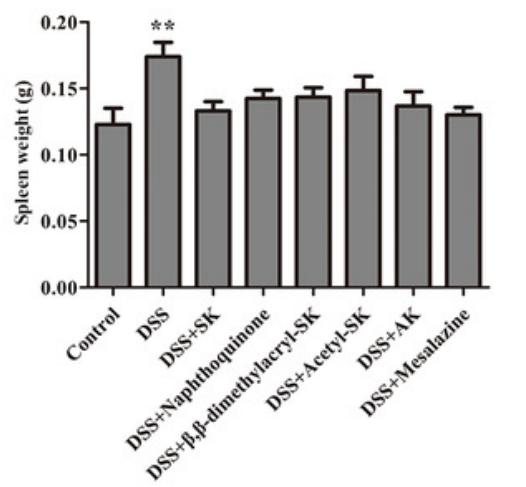




\section{Figure 2}

Colon histology and inflammation score of mice in each group (H\&E staining $200 \times$ and $400 \times)$

(A) The colon histology sections were stained with hematoxylin and eosin. The colon histology sections of DSS-treated group mice revealed sever pathology with observation of submucosal edema with extensive inflammatory cell infiltration. The frame indicates the region magnified in the bottom panel. (B) Inflammation scores of each group. Data are shown as mean \pm S.E.M, $* p<0.05, * * p<0.01$ versus control group; $\# p<0.05, \# \# p<0.01$ versus DSS-treated group ( $n=6$ per group).

(A) Control

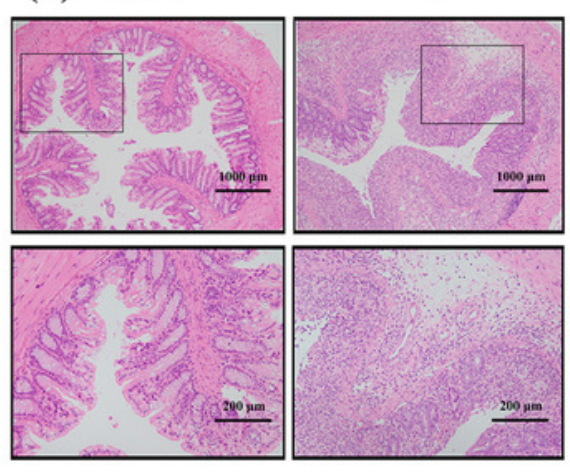

DSS+ $+\beta, \beta$-Dimethylacryl-SK

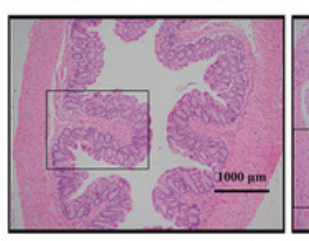

DSS+Acetyl-SK
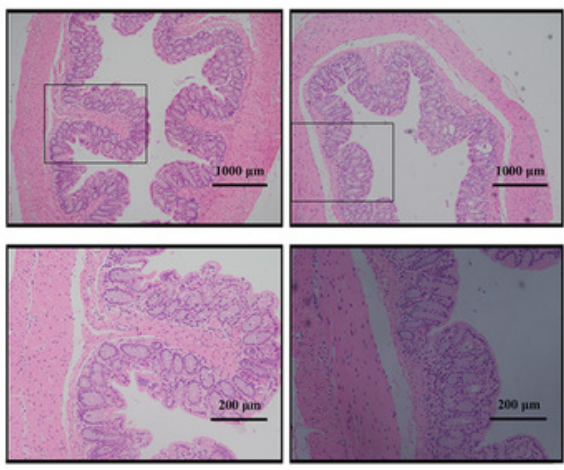

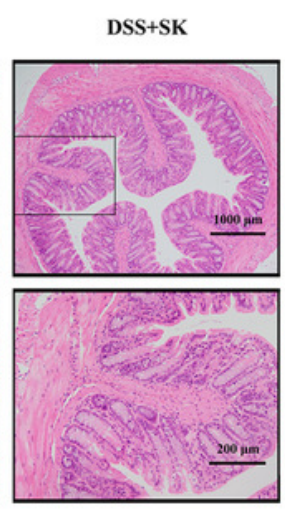

DSS+AK
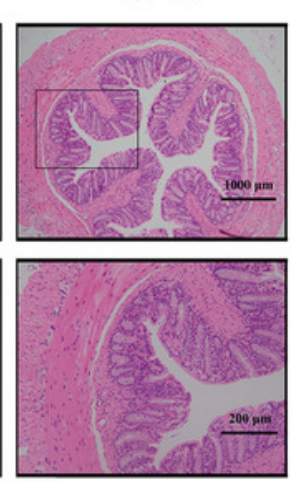

DSS+Naphthoquinone

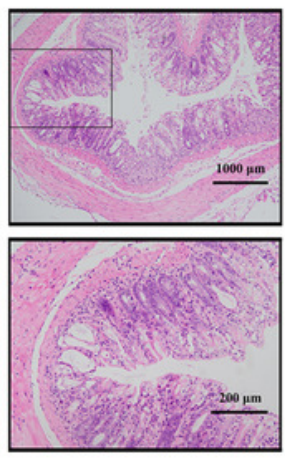

DSS+Mesalazine
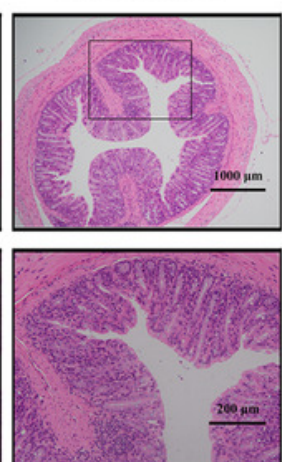

(B)
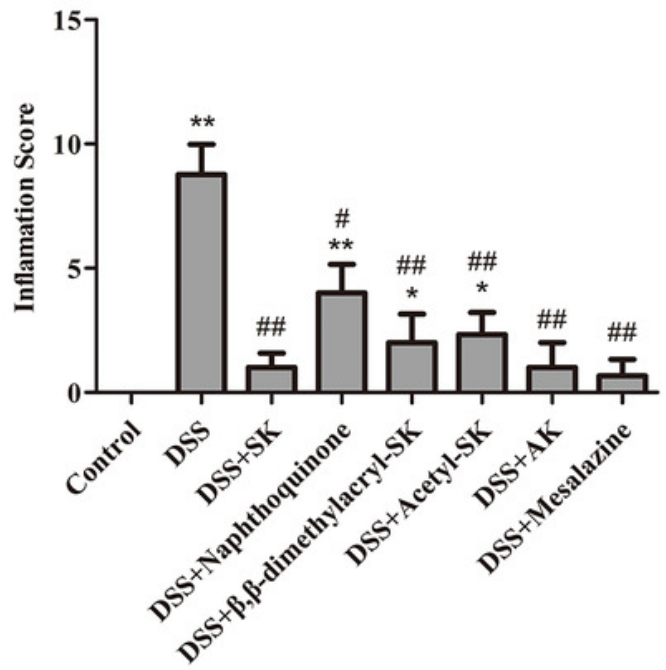
Figure 3

SK and its derivatives relieved DSS-induced colonic epithelial tight junction disruption in mice

(A) Protein expression of ZO-1, VCAM-1, Occludin and Claudin-1 in the colon tissues were analyzed by Western blot. (B) Relative protein expression ratios of were determined by densitometry and normalized to GAPDH. Each point represents the mean \pm S.E.M. from three replicates $\left({ }^{*} p<0.05,{ }^{*} p<0.01\right.$ versus control group; $\# p<0.05, \# \# p<0.01$ versus DSS-treated group).

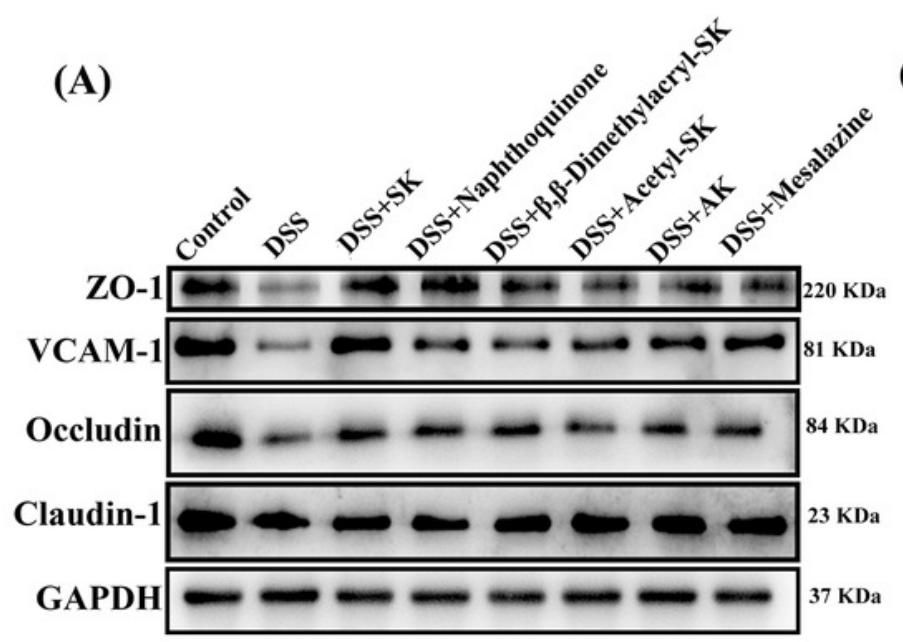

(B)

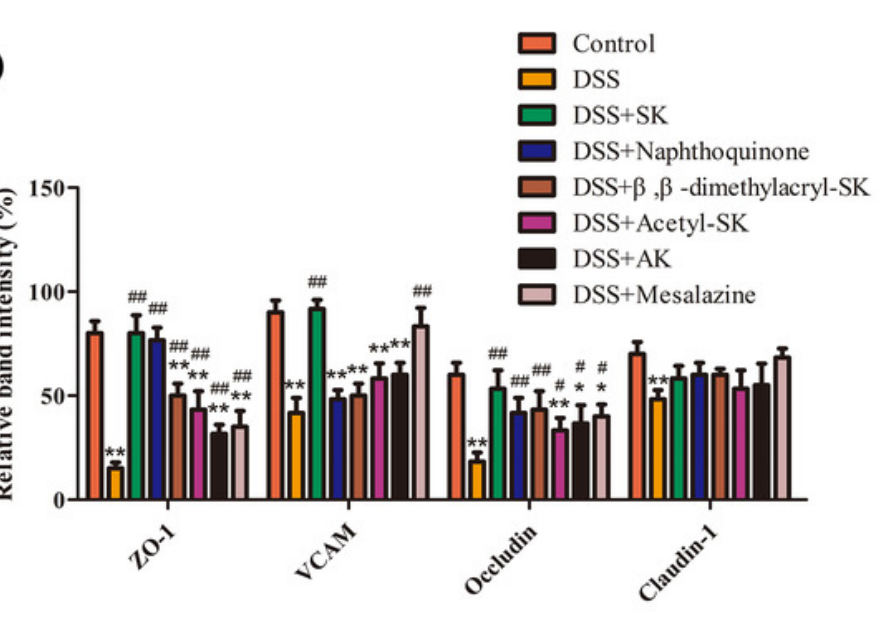


Figure 4

SK andits derivatives reduced the level of NLRP3 inflammasome activation in colonictissues

(A) Protein levels of NLRP3, ASC, caspase-1, and IL-1 $\beta$ were determined by Western blotting. $\beta$ - a ctin was used as a control. (B) Relative protein expression ratio was determined by densitometry and normalized to $\beta$ - a ctin. Each point represents the mean \pm S.E.M. from three replicates $\left({ }^{*} p<0.05,{ }^{*} p<0.01\right.$ versus control group; $\# p<0.05, \# \# p<0.01$ versus DSS-treated group).

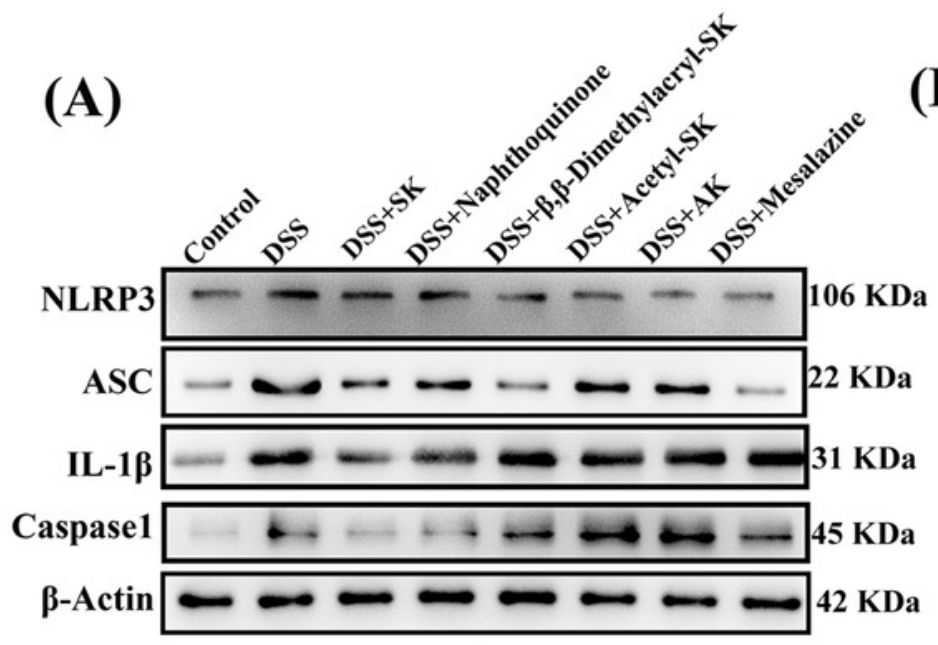

(B)

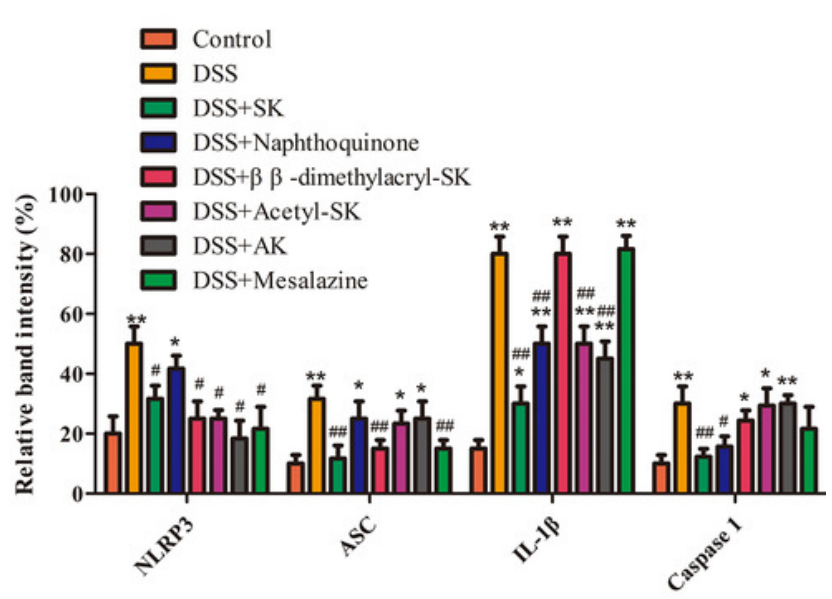




\section{Figure 5}

SK and its derivatives suppressed pro-inflammatory cytokines and promoted antiinflammatory cytokine in serum

(A-D) The protein level of cytokines IL-6, IL-1 $\beta$, TNF- $\alpha$ and IL-10 in serum were determined by ELISA (DSS: 3.5\%; SK, Naphthoquinone, $\beta, \beta$-dimethylacryl-SK, Acetyl-SK, AK: 25 mg/kg; Mesalazine: $200 \mathrm{mg} / \mathrm{kg}$ ). (E-H) Effects of different concentrations of SK on the levels of cytokines IL-6, IL-1 $\beta$, TNF- $\alpha$ and IL-10. Data are shown as mean \pm S.E.M, $* p<0.05, * * p<0.01$ versus control group; $\# p<0.05, \# \# p<0.01$ versus DSS-treated group ( $n=6$ per group).

(A)
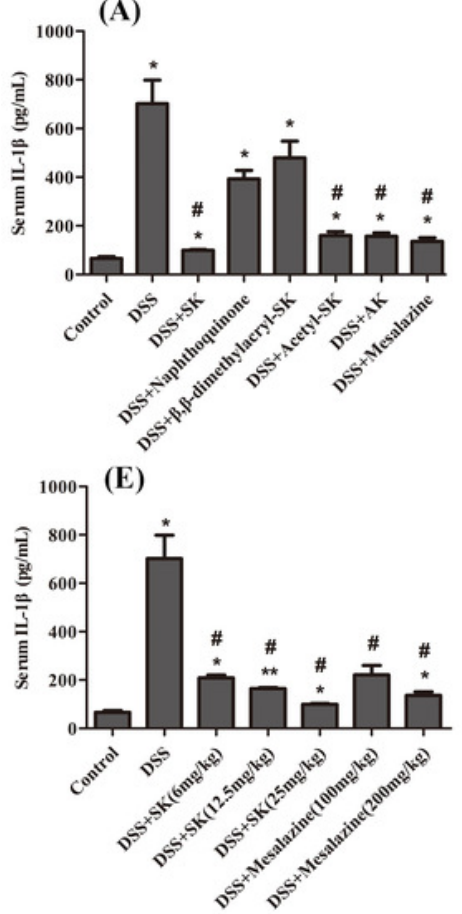

(B)
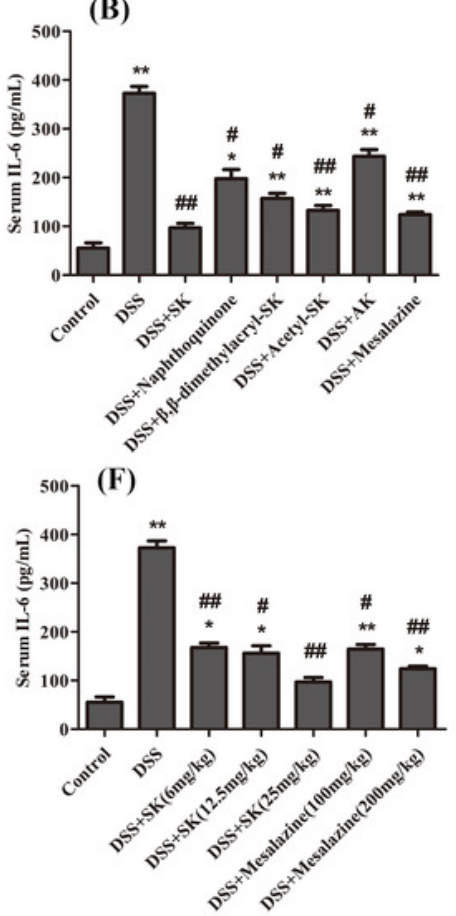

(C)

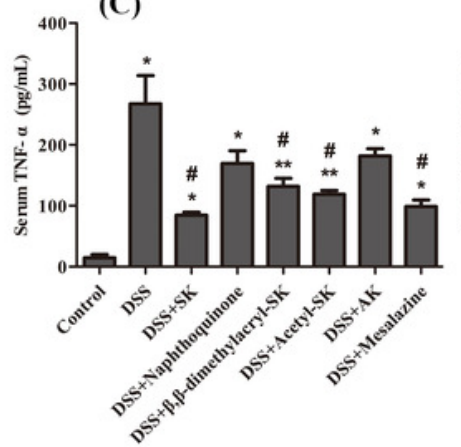

(G)

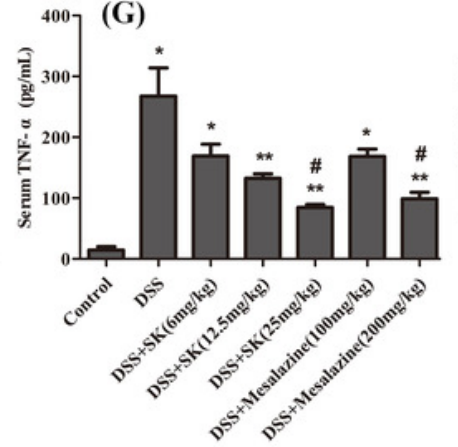

(D)
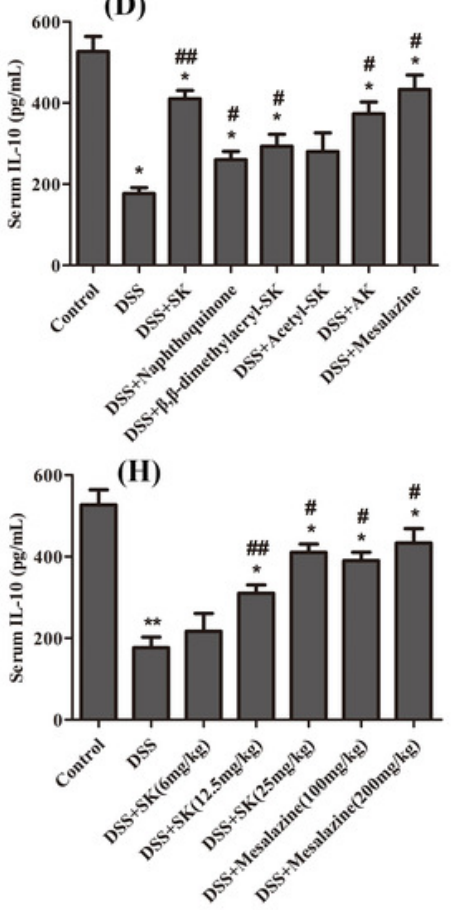


\section{Figure 6}

SK and its derivatives reduced the level of COX-2, MPO and iNOS in the serum of mice

The concentrations of COX-2 (A), MPO (B) and iNOS (C) were measured using an enzymelinked immunosorbent assay kit. Each point represents the mean \pm S.E.M. from three replicates $\left({ }^{*} p<0.05,{ }^{*} p<0.01\right.$ versus control group; $\# p<0.05, \# \# p<0.01$ versus DSS-treated group).

(A)

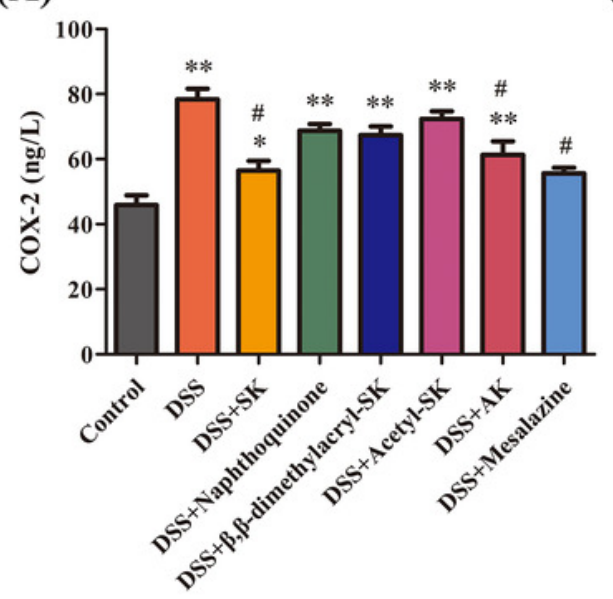

(B)

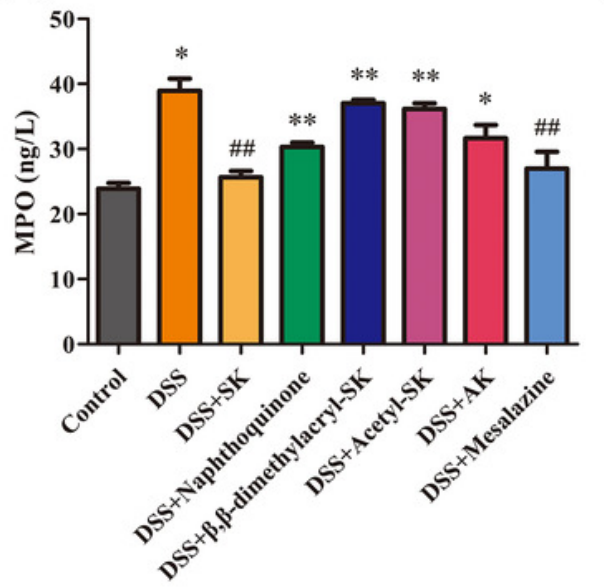

(C)

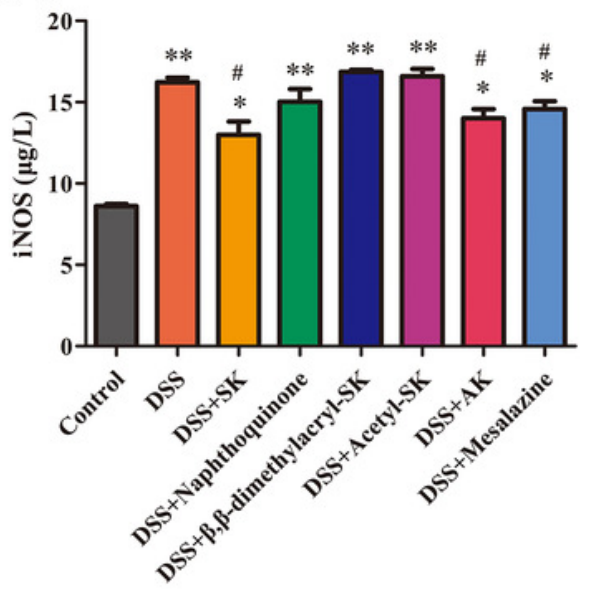




\section{Figure 7}

\section{SK andits derivatives attenuate the expression of COX-2, NF-KB and NLRP3}

(A) Immuno-histochemical analysis of COX-2 in each group. (B) Immuno-histochemical analysis of NF-KB in each group. (C) Immuno-histochemical analysis of NLRP3 in each group. The slides were incubated with primary antibody. After the secondary antibody incubation, the slides were developed with DAB and counter-stained with hematoxylin. The images were taken at $400 \times$ magnification. Data are presented as means \pm S.E.M. from three replicates $\left(* p<0.05,{ }^{* *} p<0.01\right.$ versus control group; $\# p<0.05, \# \# p<0.01$ versus DSS-treated group).

(A)

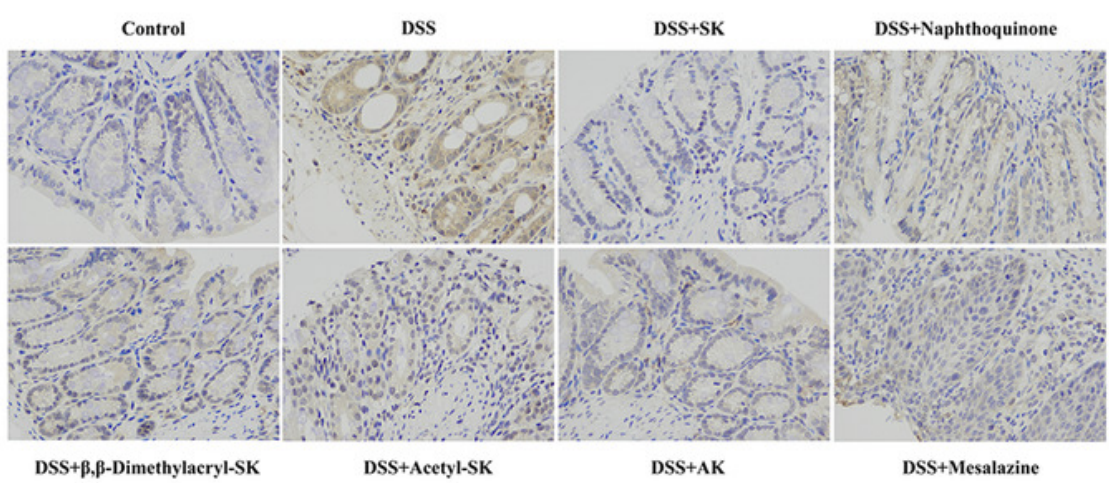

(B)

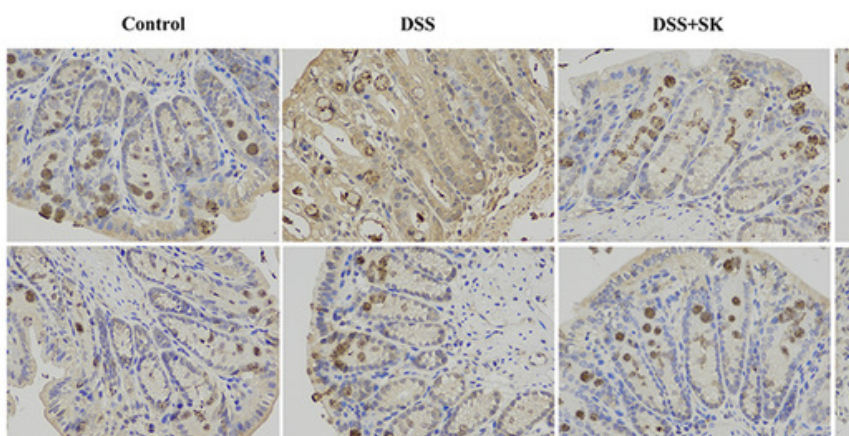

DSS+ $\beta$, $\beta$-Dimethylacryl-SK

DSS+Acetyl-SK

DSS+AK

DSS+SK

(C)

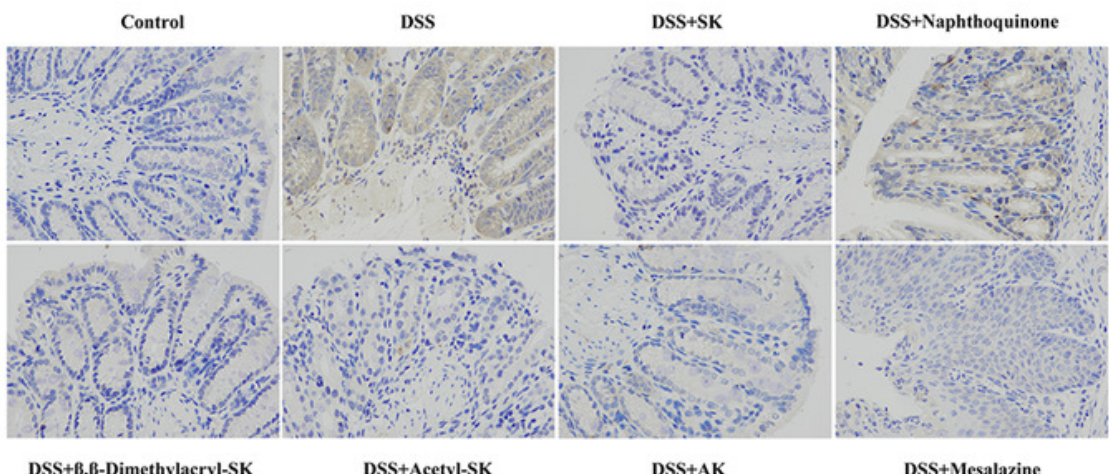

DSS+Naphthoquinone

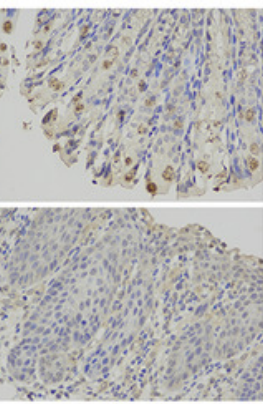

DSS+Mesalazine

DSS+Mesalazine

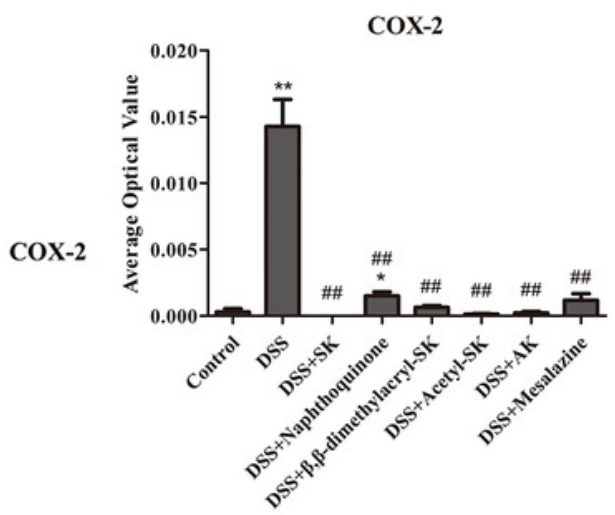

NF-K B
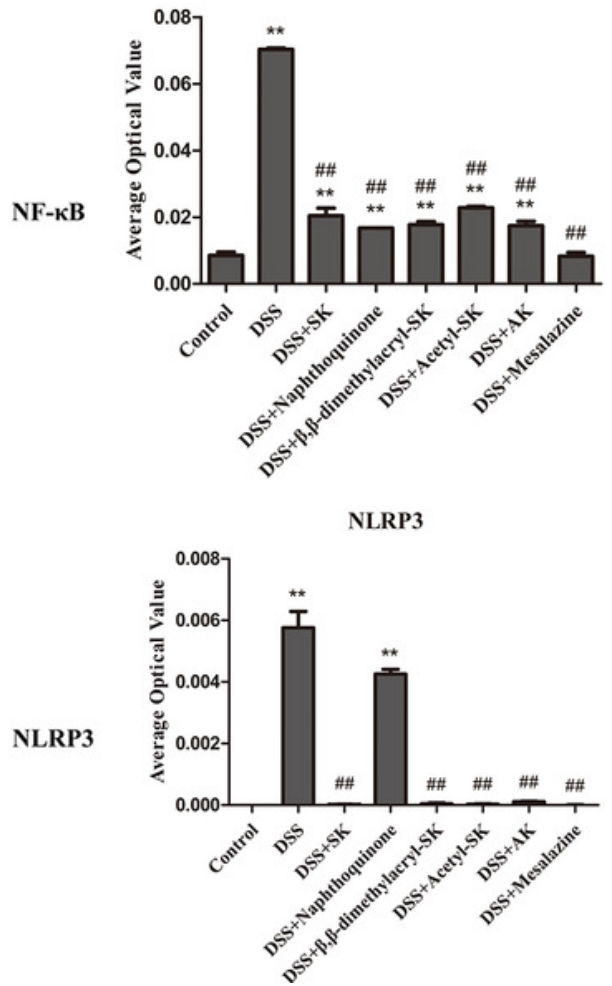


\section{Figure 8}

SK andits derivatives modulated COX-2, iNOS expression, and NF-KB signal pathway in UCmice

(A) Western blot analysis of COX-2 and iNOS levels in colonic tissues in each group. Data shown are representative of three independent experiments. (B) Relative protein expression ratios of COX-2 and iNOS were determined by densitometry. (C) Western blot analysis of proteins expression level of NF-KB signal pathway. (D) Relative protein expression ratio was determined by densitometry. Each point represents the mean \pm S.E.M. from three replicates $(* p<0.05, * * p<0.01$ versus control group;

$\# p<0.05, \# \# p<0.01$ versus DSS-treated group).

(A)

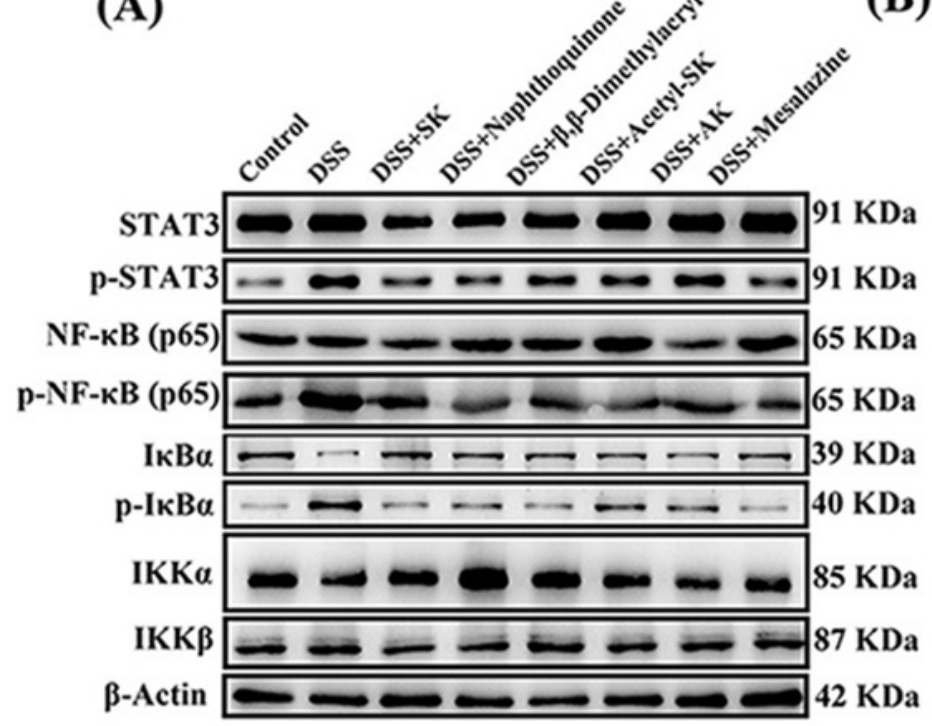

(C)

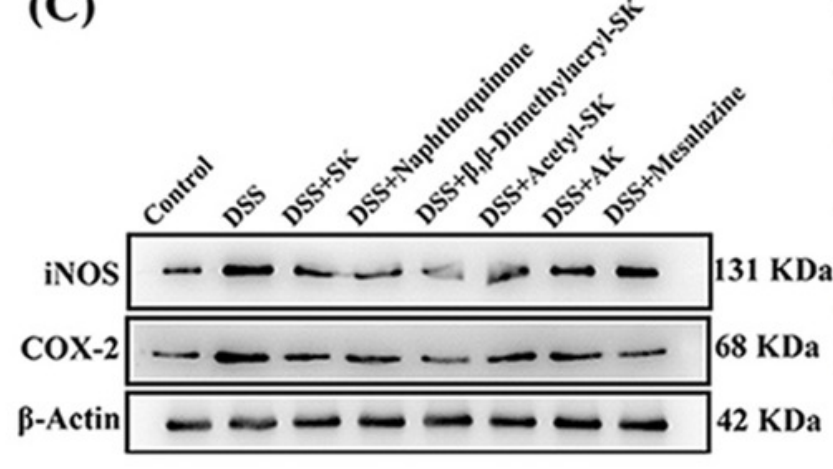

(B)

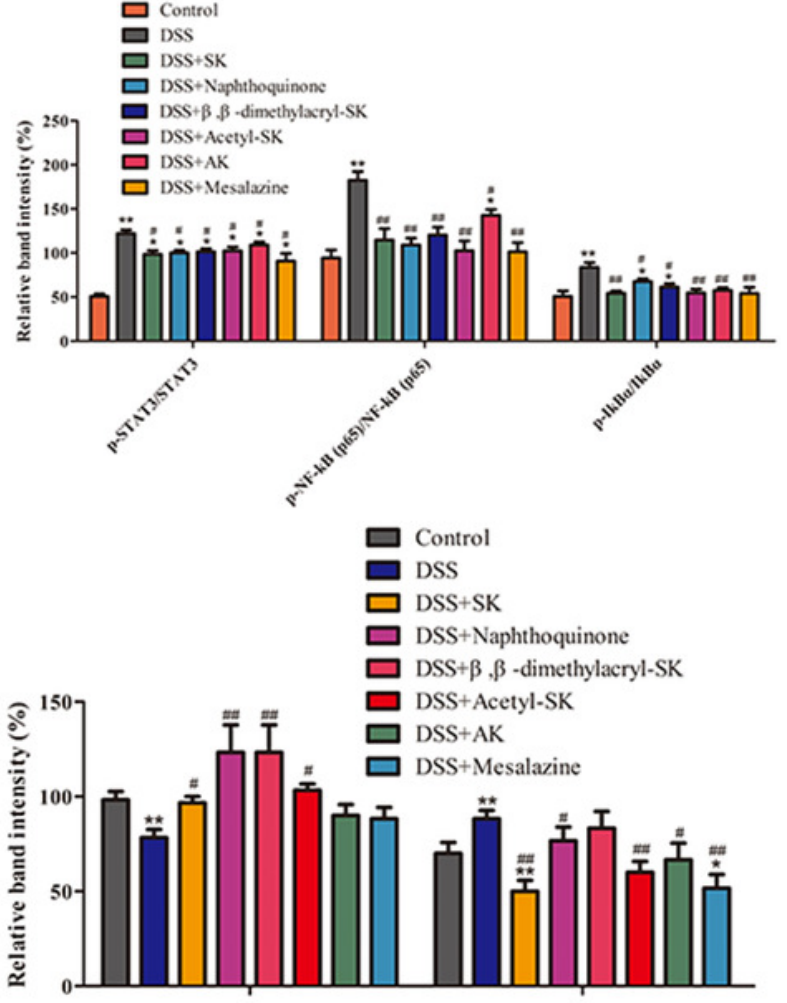

(D)

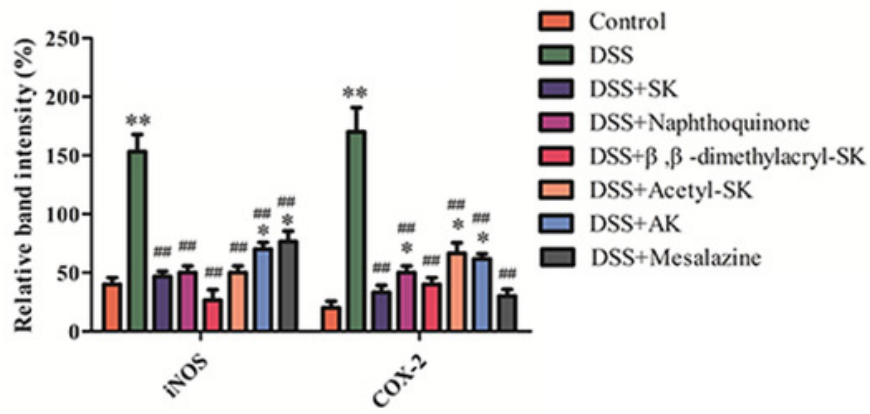

